Bundesgesundheitsbl 2012 · 55:1176-1182

DOI 10.1007/s00103-012-1533-0

Online publiziert: 29. August 2012

๑) Springer-Verlag 2012

\title{
W. Langewitz
}

Psychosomatik - Innere Medizin, Universitätsspital Basel

\section{Zur Erlernbarkeit der Arzt-Patienten-Kommunikation in der Medizinischen Ausbildung}

\section{Welche Lernziele sollen in der Arzt-Patient-Kommunikation vermittelt werden?}

Allen Versuchen einer Festlegung der Ingredienzien einer erfolgreichen professionellen Kommunikation ist gemeinsam, dass gleichzeitig eher technische Aspekte konkret beobachtbaren Verhaltens und übergreifende Einstellungen der Studierenden gegenüber Patienten angesprochen werden (z. B. [7, 8, 9]). Zum ersten Bereich gehören in den Modellen aller 3 Autorengruppen der Umgang mit Emotionen („responding to emotions“) und das Geben und Erhalten von Informationen; beides lässt sich mit geeigneten Instrumenten operationalisieren und unter standardisierten Prüfungsbedingungen auch messen (s. unten). Daneben gibt es aber in allen 3 Modellen ebenso eine Forderung, die sich mehr an die Einstellung der Studierenden gegenüber Patienten richtet: Sie sollen sich um den Aufbau einer (hilfreichen) Beziehung kümmern („building/fostering relationship“). Ganz grundlegend stellt sich allerdings die Frage, ob sich Beziehungen tatsächlich auf "bauen" lassen, was ja nahelegen würde, dass man bei Kenntnis der nötigen Bausteine gute Beziehungen wie in einem Puzzle aus geeigneten Elementen zusammensetzt. Nur dann wäre der „Aufbau“ einer hilfreichen Beziehung auch unter dem Aspekt einer Evaluation nachweisbar, indem z. B. die Häufigkeit einzelner vorab als Erfolg versprechend definierter Verhaltensweisen in einem Examen überprüft wird [10].

\section{Zur Ableitung von Lernzielen}

Auf ganz unterschiedlicher Basis ist versucht worden, Lernziele im Bereich der Arzt-Patient-Kommunikation abzuleiten (z. B. [7]). Wenn eine gute Arzt-PatientBeziehung dann gegeben ist, wenn Patienten mit einer Konsultation zufrieden sind, gäbe es kaum etwas zu verbessern an der Kommunikation, denn Patienten und $\mathrm{Pa}$ tientinnen sind in den typischen Zufriedenheitsfragebogen notorisch begeistert (z. B. [11]). Ein anderer Versuch der Begründung von Lernzielen, der allerdings nur selten versucht wurde, geht vom Feedback der Patienten aus. Ist die Beziehung dann gut, wenn Patienten Videoaufnahmen von Konsultationen als besonders gelungen bewerten? Daraus ergäben sich konkrete Lernziele in der Gesprächsführung, die zum einen kontinuierlichen Blickkontakt $[12,13]$ und zum anderen eine explizite Strukturierung des Gespräches beinhalten [14]. Wenn man allerdings eine gelungene Arzt-Patient-Beziehung unter einer umfassenderen Perspektive ansieht, z. B. als Voraussetzung für Vertrauen, sind konkrete Lernziele nicht einfach zu benennen. Die traditionelle Literatur geht davon aus, dass Vertrauen vor allem dadurch entsteht, dass Ärzte auf emotionale Äußerungen ihrer Patienten eingehen. Die prototypische Situation, an der sich dies festmachen lasse, sei das Übermitteln schlechter Nachrichten (z. B. $[15,16])$. Diese Position ist allerdings empirisch nicht belegt, gerade beim Vermitteln von schlechten Nachrichten ist nicht erwiesen, dass Patienten weniger belastet sind, wenn Ärzte ihre Emotionen explizit 
aufgreifen (z. B. $[17,18])$. Die Attraktivität und weite Verbreitung des Lernziels „Aufgreifen von Emotionen" resultiert möglicherweise aus der Ableitung erstrebenswerten kommunikativen Verhaltens aus der Psychotherapie, vor allem in der Tradition der klientzentrierten Psychotherapie von Carl Rogers (z. B. [19]) und aus dem Engagement der antiautoritären Bewegung der 1970er-Jahre für die (emotionale) Not der Unterprivilegierten, die vielen Vätern und Müttern der patientenzentrierten Kommunikation eine fundamentale moralische Orientierung vermittelte.

\section{Das explizite Ansprechen von Emotionen in der Onkologie}

Erst in den letzten Jahren ist am Beispiel von Eltern leukämiekranker Kinder und am Beispiel von Patientinnen mit Brustkrebs untersucht worden, welche Verhaltensweisen von Onkologen bzw. von Chirurgen von Betroffenen als hilfreich erlebt werden. In beiden Gruppen [20] resultieren Vertrauen und der subjektive Eindruck, emotional aufgehoben zu sein, aus der wahrgenommenen Kompetenz des Arztes und nicht aus der Bereitschaft, explizit auf Gefühle einzugehen. Patientinnen und Eltern fühlen sich emotional mit den Ärzten verbunden („emotional connection"), ohne dass jemals über Emotionen geredet wurde. Ähnliches ist für Schwangere berichtet worden, deren $\mathrm{Zu}$ friedenheit und Menge an behaltener Information mehr mit der klinischen Kompetenz von Studierenden zusammenhängt als mit der Fähigkeit, patientenzentriert mit ihnen zu sprechen - in dieser Arbeit definiert als Fähigkeit, explizit auf ihre Sorgen einzugehen [21]. Für die Lehre bedeutet dies, dass das Lernziel „Aufgreifen von Emotionen" anders begründet und wahrscheinlich auch in anderem Kontext vermittelt werden sollte; ein Beispiel ist das Aufgreifen von Skepsis oder Überraschung bei der Vermittlung von Informationen, mit denen Patienten signalisieren, dass sie "stutzen" und nicht schlicht hinnehmen, was ihnen erklärt wird (s. unten).

\section{Eine Ableitung von Lernzielen aus den Bedürfnissen der klinischen Medizin}

Bei der Neuordnung der Approbationsordnung (AO) im Jahre 2002 wurde gefordert, Studierenden vermehrt praktische Fertigkeiten, unter anderem bei der professionellen Durchführung von ArztPatient-Gesprächen zu vermitteln [22]. Dies führte zu Reformbemühungen an vielen medizinischen Fakultäten, die sich nicht nur auf den Inhalt, sondern auch auf die Vermittlungstechniken bezogen. Mittlerweile gibt es kaum noch Universitäten, die nicht typische Elemente eines ReformCurriculums anbieten. Speziell erwähnenswert sind inhaltlich die Vernetzung von vorklinischen und klinischen Inhalten und didaktisch die Reduktion von Frontalvorlesungen zugunsten des problemorientierten Lernens (PoL) sowie der Einsatz von Schauspielerpatienten. Beim PoL sollen Studierende, von einem klinischen Problem ausgehend, zunächst einmal lernen, die richtigen Fragen zu stellen und die richtigen Antworten selbstständig zu finden; der Dozent hat in diesen Gruppen vor allem koordinierende Funktion und nicht die Aufgabe, das fehlende Wissen einzubringen [23]. Schauspielerpatienten stellen bestimmte Probleme dar; in der Interaktion mit ihnen erfahren Studierende, wie verschiedene Kommunikations- oder Verhaltensweisen das Ergebnis einer Konsultation beeinflussen (s. unten; unter OSCE).

Übersichtsarbeiten zu Reform-Curricula und die persönliche Erfahrung von Lehrenden im Bereich der Arzt-PatientKommunikation belegen, dass medizinische Fakultäten sich schwertun, diesem Aufgabenbereich der Lehre ausreichend Beachtung zu schenken (z. B. [1]). Dies könnte auch damit zusammenhängen, dass die Auswahl der Lernziele und der Umfang der Lehre in professioneller Kommunikation nicht ausreichend mit Vertretern der klinischen Medizin abgesprochen wurden [24]. Dies führt in vielen Fachbereichen dazu, dass Studierende kommunikative Fertigkeiten z. B. im zweiten Studienjahr durch Dozierende der Medizinischen Psychologie oder psychotherapeutisch geschulte Dozierende und nicht von klinischen Praktikern
- z. B. aus der Inneren Medizin oder der Chirurgie - vermittelt bekommen. Damit riskiert die Lehre der Arzt-Patient-Kommunikation die Verbindung zu den klinischen Fächern zu verlieren; sie erscheint als Quantité négligeable, die mit „der richtigen Medizin“ nichts zu tun hat. Im folgenden Abschnitt wird daher versucht, Lehrinhalte der Arzt-Patient-Kommunikation aus der Perspektive klinischer Fächer zu begründen.

\section{Informationen bekommen und Informationen geben: klinische Entscheidungsfindung und informierte Patienten}

Wesentliche Entscheidungen in der klinischen Medizin basieren auf der Wahrnehmung und Integration der Phänomene, die ein Patient in der Begegnung mit einem Arzt präsentiert [25]. Diese umfassen sichtbare äußere Phänomene (z. B. veränderte Stellung von Gliedmaßen, Verfärbungen, Schwellungen) und Angaben zur Krankengeschichte. Diagnostische und therapeutische Fehler in der Inneren Medizin sind am häufigsten das Resultat vorschneller Schlüsse aus unvollständigen Daten [26]. Je präziser und umfassender anamnestische Angaben erhoben werden, desto schneller und treffender lassen sich eine weiterführende Diagnostik (z. B. Bildgebung und Labor) und allfällige therapeutische Interventionen ableiten. Das Interesse der klinischen Medizin an Kommunikationsfertigkeiten lässt sich also primär aus dem Bedürfnis ableiten, möglichst korrekte und vollständige Daten erheben zu können.

Eine zweite Aufgabe resultiert aus der Forderung, Patienten nicht mehr im Sinne eines patriarchalischen Arztbildes schlicht mit einer Verdachtsdiagnose und den weiterführenden diagnostischen Schritten zu konfrontieren, sondern sie so weit wie möglich in die ärztlichen Überlegungen einzubeziehen. Damit ist ein wesentliches Element einer patientenzentrierten Kommunikation angesprochen, deren Ziel es ist, Patienten als gut informierte Partner am Entscheidungsprozess zu beteiligen. Daher geht es um die Frage, wie Patienten so informiert werden können, dass sie wissen, auf was sie sich einlassen. In den folgenden Abschnitten werden die sich 
aus diesen Überlegungen ergebenden Lernziele kurz dargestellt, wie sie im Curriculum der Medizinischen Fakultät der Universität Basel vermittelt werden.

\section{Informationen erhalten}

Im Curriculum der Basler Medizinischen Fakultät haben diese Überlegungen dazu geführt, Kommunikationstechniken nicht mehr mit den Begriffen einer patientenzentrierten vs. arztzentrierten Kommunikation zu beschreiben, sondern die Begrifflichkeit an Kategorien der Erkenntnisgewinnung zu orientieren:

a) Studierende sind in der Lage, so zu kommunizieren, dass sie eine Hypothese formulieren und überprüfen können.

b) Studierende sind in der Lage, so zu kommunizieren, dass sie zunächst Informationen sammeln, um erste Hypothesen zur Diagnose oder zum Umgang des Patienten z. B. mit einer chronischen Erkrankung generieren zu können.

Hypothesenüberprüfendes Kommunizieren beinhaltet ge“zieltes" Nachfragen mit geschlossenen oder fokussierten Fragen; der Student weiß, wonach er sucht.

Hypothesengenerierendes Kommunizieren geschieht über ungezieltes Fragen (die typische Systemanamnese oder Fragen nach diversen Körperfunktionen) und Verhaltensweisen, die den Patienten dazu einladen, seine Geschichte zu erzählen. Dazu gehören die von uns mehrfach beschriebenen Techniken des aufmerksamen Wartens, des Wiederholens einzelner Wörter, des Spiegelns und des Zusammenfassens, die dem Patienten einen Erzählraum öffnen (WWSZ; [27]).

\section{Strukturieren}

Studierende fürchten zu Recht die Situation, dass sie von erzählfreudigen Patienten mit Material überschüttet werden, sodass sie mit ihrem Auftrag, z. B. die Hinweise auf eine koronare Herzkrankheit abzuklären, nicht fertig werden. Daher wird ihnen vermittelt, wie sie ein Gespräch strukturieren können:

a) Studierende sind in der Lage, ein Gespräch zeitlich zu planen und dies dem Patienten mitzuteilen.

Bundesgesundheitsbl 2012·55:1176-1182 DOI 10.1007/s00103-012-1533-0

C Springer-Verlag 2012

\section{W. Langewitz}

\section{Zur Erlernbarkeit der Arzt-Patienten-Kommunikation in der Medizinischen Ausbildung}

\section{Zusammenfassung}

In der vorliegenden Übersichtsarbeit werden ausgewählte Arbeiten präsentiert, die belegen, dass viele Elemente einer professionellen Arzt-Patient-Kommunikation im Medizinstudium erlernbar sind; dabei sind das Ausmaß des Lernerfolges und die Möglichkeiten seiner Überprüfung abhängig von der Definition der Lernziele. Zu ihrer Begründung wird in den meisten Lehrplänen auf die Bedeutung eines eher partnerschaftlichen und nicht patriarchalischen Umgangs mit Patienten verwiesen. Dem wird hier eine Definition von Lehrzielen gegenübergestellt, die sich an den Bedürfnissen der klinischen Medizin orientiert: Studierende erheben belastbare anamnestische Angaben, sie können Gespräche explizit strukturieren und Informationen vermitteln, sie trauen sich zu, emotionale Äußerungen von Patienten aufzugreifen. Als Prüfungsformat in der professionel- len Kommunikation wird das Objective Standardised Clinical Examination (OSCE) mit seinen Stärken und Schwächen diskutiert. In Zukunft wird der strukturierte Einsatz von Videoaufnahmen von Gesprächen dazu führen, dass Studierende das eigene Verhalten besser kritisch reflektieren können. Wünschenswert wäre der Einsatz von Verfahren zur unmittelbaren Rückmeldung an Studierende über arbeitsplatzbasierte Techniken wie MiniCex oder Workplace-based Assessment. Diese Lehr- und Prüfungsmethoden bedürfen allerdings einer nicht nur fachlich, sondern auch in der Kunst des Feedback-Gebens ausgebildeten Fakultät.

Schlüsselwörter

Arzt-Patient-Kommunikation - Lernziele . Klinische Medizin · OSCE · Video-Feedback

\section{Physician-patient communication in medical education: can it be learned?}

\section{Abstract}

Based on a review of recent key articles, this paper demonstrates that many elements of physician-patient communication can be learned successfully during medical education. Methods of assessment and definition of success depend largely on the definition of teaching goals, which are usually based on the principles of a more egalitarian and nonpaternalistic physician-patient communication. In this article another approach is suggested. Teaching objectives in patient-physician communication can also be deduced from the needs of clinical medicine, resulting in the following goals: students are able to gather relevant data from patients' history, they explicitly structure the consultation and the way they give information, they know how to respond to patients' emotions. The Objective Standardised Clinical Examination (OSCE) is discussed with its strengths and weaknesses. The inclusion of videobased feedback is presented as a teaching tool to improve students' self-reflection. Workplace-based assessment and Mini-CEX are promising educational tools that require a well-trained faculty, not only in the teaching and practice of communication but also in the art of giving constructive and yet honest feedback.

Keywords

Physician-patient communication · Teaching goals · Clinical medicine $\cdot$ OSCE V Video feedback b) Studierende sind in der Lage, ein Gespräch thematisch einzugrenzen und den Patienten ggf. auf ein bestimmtes Thema zurückzuführen.

c) Studierende können den Erzählraum explizit eingrenzen und erweitern.

Diese Lernziele lassen sich unter dem Begriff des expliziten Strukturierens zusam- menfassen, das sich auf Zeitangaben („Ich soll innerhalb der nächsten $20 \mathrm{~min}$..."), Festlegen eines Themas („... herausfinden, wie sich bei Ihnen die Herzerkrankung entwickelt hat") und Mitteilen der eigenen Funktion (,... so, als ob ich der Stationsarzt wäre, der Sie gestern aufgenommen hat") bezieht [27]. 


\section{Vermitteln von Informationen}

Ärzte und Studierende vermitteln Informationen in der Regel unstrukturiert; Studierende folgen der Logik ihrer vom Aufbau des Medizinstudiums geprägten Lernstrategie (z. B. entlang eines pathophysiologisch begründeten roten Fadens), Ärzte folgen dem Ablauf ihres eigenen Handelns (ihre Erklärungen folgen dem Weg der Katheterspitze von der Leiste zur linken Herzkammer). Beide Ordnungsschemata sind weit entfernt von Laienkonzepten und zudem nur implizit rekonstruierbar, da sie nicht offen deklariert werden. Daraus ergibt sich für die Vermittlung von Information die Forderung:

a) Studierende können Informationen strukturiert vermitteln und diese Struktur zu Beginn ihrer Erklärungen explizit ansprechen.

b) Studierende sind in der Lage, Informationen in so kleinen Einheiten zu vermitteln, dass sie sicher sind, dass Patienten ihren Erklärungen aufmerksam folgen.

c) Studierende können sich Rechenschaft darüber geben, was Patienten verstanden haben.

Wir haben für die explizite Vermittlung von Struktur in der Informationsvermittlung den Begriff der Buchmetapher entwickelt: Genauso wie ein Buch seinen Inhalt in Überschrift, Inhaltsverzeichnis, Kapitel und Text gliedert, sollte medizinische Information in unterschiedlichen Segmenten mit eindeutiger Zuordnung vermittelt werden [27].

Innerhalb des Themas der Informationsvermittlung lassen sich der Einsatz von Pausen und das Aufgreifen von Emotionen als kommunikative Techniken verdeutlichen und im Rollenspiel konkret üben. Nur dann, wenn Studierende nach einer Information eine Pause machen, den Patienten dabei anschauen und warten, bis er durch Nicken oder „hmm“ seine Bereitschaft zur Fortsetzung signalisiert, können sie sicher sein, dass sie ungeteilte Aufmerksamkeit haben.

In diesen Momenten der Abstimmung zwischen Patient und Student artikulieren Patienten (und nach entsprechendem Training auch simulierte Patienten) Skepsis oder Beunruhigung, die dann durch
Ansprechen der Emotion aufgegriffen und im nächsten Schritt geklärt wird.

\section{Aufgreifen von Emotionen}

Emotionale Äußerungen kommen in typischen Gesprächssituationen kaum vor (z. B. [28]; über Abklärungsgespräch in einer medizinischen Ambulanz), intensive Emotionen wie tiefe Verzweiflung oder wütender Protest sind seltene Ausnahmen. Dennoch sollten Studierende über ein Werkzeug verfügen, mit emotionalen Äußerungen hoher Intensität umzugehen, damit sie nicht aus Angst vor der eigenen Inkompetenz bei diskreten Anzeichen von Gefühlen des Patienten abrupt auf die Sachebene wechseln. Eine Mischung unterschiedlicher Gesprächstechniken zum Umgang mit Emotionen ist von Back [29] unter dem Akronym NURSE vorgestellt und evaluiert worden, dem wir in Basel in der Ausbildung von Studierenden folgen:

- Studierende sind in der Lage, die Elemente des NURSE-Modells beim Umgang mit emotionalen Äußerungen des Patienten einzusetzen.

Unter diesem Akronym verbergen sich folgende Elemente:

- Naming emotion: das explizite Ansprechen von Gefühlen,

- Understanding emotion: (wenn möglich) Verständnis äußern für Gefühle des Patienten,

- Respecting: dem Patienten vermitteln, dass seine Gefühle oder sein Umgang mit Gefühlen Respekt verdient,

- Supporting: dem Patienten beim Umgang mit belastenden Gefühlen Unterstützung anbieten,

- Exploring emotion: nicht eindeutige oder nachvollziehbare Stimmungen ansprechen und klären [27].

Bei intensiven Emotionen besteht das größte Problem für Studierende im Aushalten der Pause, in der der Patient um seine Fassung ringt. Diese Pause ist einfacher zu ertragen, wenn Studierende wissen, wie sie selber den Kontakt zum Patienten wieder aufnehmen können: durch das Ansprechen von Gefühlen oder aber, wenn diese bereits eindeutig geäußert wurden, durch Vermitteln ihres Verste- hens („Ich kann gut verstehen, dass Sie verzweifelt sind“).

\section{Überprüfung und Evaluation von Lernzielen im Bereich der professionellen Kommunikation}

Eine umfangreiche Literatur berichtet über die verschiedenen Möglichkeiten, wie man kommunikative Kompetenzen messen und überprüfen kann. Dabei versteht sich von selbst, dass die Ausgestaltung der Prüfungsformate und die Definition der abhängigen Variablen auf die Lernziele des Unterrichts ausgerichtet sind $[30,31,32]$.

Arzt-Patient-Kommunikation ist im Wesentlichen eine Kompetenz, die sich im Handeln zeigt und weniger im Wissen um theoretische Konzepte [33]. Daher lässt sich der Erwerb kommunikativer Fertigkeiten nicht in klassischen wissensbasierten Multiple-Choice-Prüfungen evaluieren, sondern eher über die Beobachtung konkreten Verhaltens unter standardisierten Bedingungen. Typisches Instrument ist der OSCE, der im Folgenden vorgestellt wird.

\section{Objective Structured Clinical Examination (OSCE)}

Die meisten Universitäten, die im Rahmen ihrer Reformbemühungen klinische Fertigkeiten und unter ihnen die Arzt-Patient-Kommunikation vermitteln, überprüfen das Erreichen der entsprechenden Lernziele mit diesem Prüfungsformat. In der Regel begegnet der Studierende im OSCE einem standardisierten Patienten, der bestimmte Befunde oder eine spezifische Anamnese präsentiert. Für die Gesprächsführung wird bewertet, wie der Student mit dem Patienten kommuniziert, ob z. B. bestimmte Verhaltensweisen, die vorher als Lernziel definiert und vermittelt wurden, im Kontakt mit dem simulierten Patienten gezeigt werden. Typische Beispiele sind:

- Der Student stellt sich mit Namen und Funktion vor, er benennt die Zeitgrenzen der (Prüfungs-)Konsultation.

- Der Student leitet explizit von der Erhebung des jetzigen Leidens (hypothesengenerierende Kommunikation, 
s. oben) auf die Klärung der Differenzialdiagnose über (hypothesenüberprüfende Kommunikation).

- Der Student wartet, bis der Patient von selbst beginnt zu sprechen (bei der Prüfungsaufgabe „Vermitteln schlechter Nachrichten“).

- Der Student leitet die Vermittlung von Informationen mit einer expliziten Struktur ein (Buchmetapher, s. oben).

Strittig ist bei der Auswertung von OSCEs, ob eher konkrete Verhaltensweisen bewertet werden sollen oder ob Globalskalen angemessen sind. In der Diskussion werden zum einen methodische Argumente zur Reliabilität des Expertenratings mit diesen Skalentypen angeführt (z. B. [34, 35, 36, 37, 38, 39]), zum anderen geht es um die Frage, mit welchem Aufwand Examinatoren geschult werden müssten, um zuverlässig die Prüfungsleistungen von Studierenden mit dem einen oder anderen Evaluationsinstrument beurteilen zu können.

Die oben beispielhaft aufgeführten Elemente von Checklisten gehören in den Bereich der konkreten, im Einzelnen beschriebenen Verhaltensweisen, die vor allem dann sinnvoll sind, wenn in der Lehre genau diese Fertigkeiten gründlich geübt werden.

Eine typische Prüfung unter den Bedingungen eines OSCE dauert pro Station 7-15 min, jeder Student durchläuft mehrere OSCE-Stationen, in denen unterschiedliche Fertigkeiten überprüft werden.

\section{Typische Probleme des OSCE}

Ein besonderes Problem des OSCE im Bereich der Arzt-Patient-Kommunikation ist die Ausbildung der simulierten Patienten, die möglichst allen Studierenden gleiche anamnestische Angaben zur Verfügung stellen sollten. Damit lässt sich aber nicht überprüfen, ob ein Student in der Lage ist, den Patienten ins Erzählen zu bringen - dies würde nämlich dazu führen, dass simulierte Patienten unterschiedliche Mengen an Information preisgeben, abhängig von der Qualität der hypothesengenerierenden Kommunikation des Studenten. Aus diesem Dilemma wird oft die Konsequenz gezogen, simulierte Patienten nach einer initialen Äußerung zum Schweigen zu verpflichten und weitere anamnestische Angaben nur noch auf Fragen hin geben zu lassen. Der typische OSCE benachteiligt also die Studierenden, die gelernt haben zuzuhören und begünstigt diejenigen, die vorbereitete Listen von Fragen schematisch abarbeiten; damit lässt sich dann bestenfalls das hypothesenüberprüfende Kommunizieren des Studenten überprüfen. Ein möglicher Ausweg aus diesem Dilemma besteht darin, nicht nur die Einleitungssätze zu standardisieren, z. B.: „Ich habe seit einigen Monaten Schmerzen in den Beinen, wenn ich meine Tochter besuchen will und drei Stockwerke nach oben steigen muss", sondern auch die zusätzlichen Informationen, die Schauspieler-Patienten mitteilen würden, wenn der Student sie durch Warten oder Wiederholen einzelner Worte (,in den Beinen ...?") dazu ermuntert fortzusetzen: „Ja, das wird immer schlimmer in letzter Zeit; auch wenn ich schneller laufen will, zum Beispiel auf's Tram rennen, merke ich das. Wenn ich dann stehen bleibe, wird's langsam besser."

Ein weiteres Problem dieses Prüfungsformates ist die Tendenz der Studierenden, gewünschte Verhaltensweisen der Kommunikation, z. B. die Elemente des NURSE-Modells, in floskelhafter Manier auswendig zu lernen und dann in der Prüfung zu reproduzieren [40]. Letztlich wäre die erstaunliche Fähigkeit der Studierenden, sich an Prüfungsbedingungen anzupassen, nur dann zu konterkarieren, wenn jedes Jahr neue Anforderungen formuliert würden - eine auch in wissensbasierten Prüfungen unmögliche Forderung. Im Bereich kommunikativer Fertigkeiten wird versucht, diesem Dilemma dadurch zu begegnen, dass Checklisten konkreten Verhaltens mit Globalbeurteilungen kombiniert werden, die den Prüfer z. B. auffordern, sich festzulegen, ob „Informationen in einer Art und Weise vermittelt wurden, die dem Auffassungsvermögen des Patienten angemessen ist“. Wahrscheinlich ist also der kombinierte Einsatz von Checklisten und Global Ratings der beste Ansatz [40]. Dennoch bleibt das Problem, dass gerade im Bereich der Beziehungsgestaltung, bei der es um Authentizität und affektives Mitschwingen geht, Prüfungen unter Einbezug simulierter Patienten, die eben nur so tun, als seien sie traurig, und die mit Studierenden interagieren, die nur so tun, als seien sie betroffen, kaum möglich sind [41, 42]. Allerdings gibt es auch Übersichtsarbeiten, die zu eher optimistischeren Schlussfolgerungen kommen: Es sei im Prinzip möglich, die emotionale Intelligenz von Studierenden durch geeignete Intervention zu verbessern und dies in Gesprächen mit simulierten Patienten abzubilden [43].

\section{Ausmaß des Erfolges der Lehre im Bereich der Arzt-Patient-Kommunikation}

Außerhalb des Studentenunterrichts kommen Übersichtsartikel zum Schluss, dass sich Techniken der patientenzentrierten Kommunikation erfolgreich vermitteln lassen [44]. Zu einzelnen Fertigkeiten liegen Arbeiten vor; als Beispiel sei die gründliche Arbeit von Schildmann et al. genannt, in der mit unterschiedlichen methodischen Ansätzen gezeigt wird, dass sich das Überbringen schlechter Nachrichten erfolgreich vermitteln lässt [45]. Grundsätzlich ist der Stand der empirischen Forschung allerdings nicht befriedigend [46, 47]. Das Fehlen gut abgesicherter Daten zum Vergleich unterschiedlicher didaktischer Zugänge zur Vermittlung von Lernzielen in der Humanmedizin hat zur Gründung der „Best Evidence in Medical Education (BEME) Collaboration" [48] geführt, einer Institution, die sich darum bemüht, ähnlich der Cochrane-Bibliothek, Evidenz für erfolgreiche Interventionen in der Lehre zusammenzutragen und Qualitätskriterien für Studien im Bereich der Lehre festzulegen [49].

Die bisher größte prospektive Studie zu den Langzeitfolgen guter kommunikativer Fertigkeiten wurde 2007 von Tamblyn vorgestellt [50]. Die Autoren zeigen, dass schlechte Abschlussnoten im Staatsexamen im Bereich Arzt-Patient-Kommunikation vorhersagen, gegen wen in den nachfolgenden Jahren Beschwerden von Patienten aktenkundig werden.

Nicht spezifisch für den Erwerb kommunikativer Fertigkeiten, sondern generell für den Erfolg eines Reform-Curriculums, das praktische Fertigkeiten vermit- 
telt, ist die Arbeit von O'Neill et al. [51]. In ihr wird berichtet, dass sich Absolventen eines Reform-Curriculums im Vergleich $\mathrm{zu}$ Absolventen eines traditionellen Curriculums besser selbst einschätzen können, rechtzeitig Hilfe holen und besser in der Lage sind, mit Unsicherheit umzugehen.

\section{Neue Medien und Lehre in der Arzt-Patient-Kommunikation}

Wenn man davon ausgeht, dass sich Fertigkeiten in der Arzt-Patient-Kommunikation vor allem in der Praxis erwerben lassen und bewähren müssen, also im direkten Kontakt mit simulierten oder echten Patienten, dann sind webbasierte Interventionen und ähnliche didaktische Instrumente nur mittelbar einzusetzen. Allerdings fördern sie möglicherweise die Selbstwahrnehmung von Studierenden, indem sie ihnen ermöglichen, sich selbst in Interaktion zu erleben und kritisch zu hinterfragen.

Eine Möglichkeit ist die Arbeit mit Videos von eigenen Gesprächen [52, 53], in denen Studierende bestimmte a priori definierte Fertigkeiten identifizieren und sich dazu äußern sollen, warum sie diese Techniken einsetzen, welche Auswirkungen dies hatte und welche Alternativen ihnen einfallen. In einem zweiten Schritt ließe sich die Fähigkeit der Studierenden, bestimmte Kommunikationstechniken und ihre Auswirkungen zu identifizieren, verbessern, indem sie ihre eigene Auswertung mit einem Experten-Rating vergleichen. Gerade im Bereich der Informationsvermittlung lässt sich so zeigen, dass Studierende die Menge an Informationen, die sie bei der Aufklärung über einen Eingriff im Rollenspiel vermitteln, deutlich unterschätzen; mithilfe ihres fachlichen Wissens verknüpfen sie einzelne Elemente zu übergeordneten Informationseinheiten und erkennen nicht mehr, dass sie damit Laien überfordern.

Das Identifizieren von Kommunikationstechniken in Videoclips lässt sich auch als eine Variante des OSCE einsetzen, indem Studierende in der Prüfung aufgefordert werden, diese Techniken zu benennen oder fehlerhaft eingesetzte Techniken zu identifizieren (z. B. Benennen einer Emotion ohne Abwarten einer Reaktion vom Patienten; Zusammenfas- sen der Anamnese ohne vorherige Ankündigung). Allerdings zeigt eine aktuelle Arbeit statistisch signifikante, aber letztlich marginale Verbesserungen in den Examina von Studierenden, die sich zur Vorbereitung eine 90-minütige Dokumentation guten klinischen Verhaltens anschauen [54].

Ein weiteres Argument dafür, Studierenden systematisch Videomaterial ihrer Interaktionen mit Patienten zur Verfügung zu stellen, lässt sich daraus ableiten, dass Patienten eher selten explizit Feedback zu den Kommunikationsfertigkeiten ihrer Ärzte geben, geschweige denn den Studierenden. Für das Lernen am Erfolg oder Misserfolg des eigenen Handelns fehlt also häufig ein zentrales Element: die Rückmeldung des betroffenen Gegenübers. Dies ist beim Erlernen anderer praktischer Fähigkeiten einfacher: Die Venenpunktion, das Identifizieren sensibler Ausfälle oder die korrekte und nicht unnötig belastende körperliche Untersuchung des Oberbauches bei einer Cholezystitis lassen sich über die Reaktionen des Patienten verifizieren.

Wahrscheinlich ist, dass Studierende am meisten von einer Intervention profitieren, die unmittelbar auf ihr Handeln folgt [55]. Vor allem für Ärzte in den ersten Jahren ihrer Facharztausbildung sind mit dem Mini-Cex oder der arbeitsplatzbasierten Evaluation von Fertigkeiten entsprechende Instrumente entwickelt worden [56], deren methodische Zuverlässigkeit aber (noch) nicht so ausgereift ist, dass sie im Bereich der Lehre summative Prüfungen unter standardisierten Bedingungen wie im OSCE ersetzen würde.

Am Beispiel des unmittelbaren Feedbacks „bei der Arbeit“ („workplace based assessment") lässt sich am ehesten ein kritisches Element der Lehre im Bereich der Arzt-Patient-Kommunikation konstatieren: Eine solche Form der Lehre ist personalintensiv, und sie fordert Lehrende, die nicht nur die Techniken der professionellen Kommunikation mit Patienten beherrschen, sondern auch eine fördernde Haltung gegenüber Studierenden einzunehmen bereit sind - hier begegnet uns also in der Weiterbildung des Lehrkörpers das gleiche Problem wie in der Ausbildung der Studierenden: Technik und
Haltung gilt es zu schulen und zu entwickeln, „Herz und Hand“ müssen gleichermaßen erreicht werden.

\section{Fazit}

Professionelle Arzt-Patient-Kommunikation geht über den gesunden Menschenverstand und wohlerzogenes Verhalten hinaus. Wesentliche Elemente der ärztlichen Gesprächsführung sind als konkrete Lernziele eindeutig definiert, es stehen Methoden zur Verfügung, um diese Lernziele zu vermitteln, und Prüfungsformate, um ihr Erreichen zu messen. Dieser Bereich der ärztlichen Professionalität wird in der Lehre von neuen Verfahren profitieren, bei denen Studierende konkretes Feedback auf eigenes Verhalten bekommen und zur Selbstreflexion angeregt werden. Dies setzt allerdings voraus, dass es in den Fakultäten genügend klinische Dozenten gibt, die sich mit diesen Lehrformen vertraut machen und selbst über eine hohe professionelle Kompetenz im Bereich der Arzt-PatientKommunikation verfügen.

\section{Korrespondenzadresse}

Prof. Dr. W. Langewitz

Psychosomatik - Innere Medizin,

Universitätsspital Basel

Hebelstr. 2, 4031 Basel

Schweiz

wlangewitz@uhbs.ch

Interessenkonflikt. Der korrespondierende Autor gibt an, dass kein Interessenkonflikt besteht.

\section{Literatur}

1. Hargie O, Boohan M, McCoy M, Murphy P (2010) Current trends in Communication Skills Training in UK schools of medicine. Med Teach 32:385-391

2. Swing SR (2007) The ACGME outcome project: retrospective and prospective. Med Teach 29:648654

3. Kiessling C, Dieterich A, Fabry G, Hölzer H, Langewitz W, Mühlinghaus I, Pruskil S, Scheffer S, Schubert S (2010) Communication and social competencies in medical education in German-speaking countries: the Basel consensus statement. Results of a Delphi survey. Patient Education Couns 81:1259-1266

4. Rubin P, Franchi-Christopher D (2002) New edition of tomorrow's doctors. Med Teach 24:368-369

5. Frank JR, Danoff D (2007) The CanMEDS initiative: implementing an outcomes-based framework of physician competencies. Med Teach 29: 642-647 
6. Albanese MA, Mejicano G, Anderson WM, Gruppen $L$ (2010) Building a competency-based curriculum: the agony and the ecstasy. Advances Health Sci Education: Theory Practice 15:439-454

7. de Haes H, Bensing J (2009) Endpoints in medical communication research, proposing a framework of functions and outcomes. Patient Education Couns 74:287-294

8. Lazare A, Putnam SM, Lipkin M (1995) Three functions of the medical interview. In: Lazare A, Putnam SM, Lipkin M (Hrsg) The medical interview. Clinical care, education and research. Springer, New York Heidelberg

9. Epstein RM, Street RL (2007) Patient-centered communication in cancer care: promoting healing and reducing suffering. In: NIH Publication No. 076225. National Cancer Institute, Bethesda, MD

10. Salmon P, Young B (2005) Core assumptions and research opportunities in clinical communication. Patient Education Couns 58:225-234

11. Langewitz W, Keller A, Denz M, Wossmer-Buntschu B, Kiss A (1995) Patientenzufriedenheits-Fragebogen (PZF): Ein taugliches Mittel zur Qualitätskontrolle der Arzt-Patient-Beziehung? Psychother Psychosom Med Psychol 45:351-357

12. Gorawara-Bhat R, Cook MA (2011) Eye contact in patient-centered communication. Patient Education Couns 82:442-447

13. Marcinowicz L, Konstantynowicz J, Godlewski C (2010) Patients' perceptions of GP non-verbal communication: a qualitative study. Br J Gen Pract 60:83-87

14. Robinson J, Walley T, Pearson M, Taylor D, Barton $S$ (2002) Measuring consultation skills in primary care in England: evaluation and development of content of the MAAS scale. Br J Gen Pract 52:889893

15. Del Piccolo L, de Haes H, Heaven C, et al (2011) Development of the Verona Coding Definitions of Emotional Sequences to code health providers' responses (VR-CoDES-P) to patient cues and concerns. Patient Education Couns 82:149-155

16. Zimmermann C, Del Piccolo L, Bensing J et al (2011) Coding patient emotional cues and concerns in medical consultations: The Verona Coding Definitions of Emotional Sequences (VR-CoDES). Patient Education Couns 82:141-148

17. Butow PN, Brown RF, Cogar S, Tattersall MH, Dunn SM (2002) Oncologists' reactions to cancer patients' verbal cues. Psychooncology 11:47-58

18. Barth J, Lannen P (2010) Efficacy of communication skills training courses in oncology: a systematic review and meta-analysis. Annals Oncol 22:1030-1040

19. Bird J, Cohen-Cole SA (1990) The three-function model of the medical interview. An educational device. Adv Psychosom Med 20:65-88

20. Young B, Ward J, Forsey M, Gravenhorst K, Salmon $\mathrm{P}$ (2011) Examining the validity of the unitary theory of clinical relationships: comparison of observed and experienced parent-doctor interaction. Patient Education Couns 85:60-67

21. O'Keefe $M$, Roberton $D$, Sawyer $M$, Baghurst $P$ (2003) Medical student interviewing: a randomized trial of patient-centredness and clinical competence. Family Practice 20:213-219

22. Approbationsordnung für Ärzte, ausgegeben zu Bonn am 3. Juli 2002. Bundesgesetzblatt Jahrgang 2002 Teil I Nr. 44, 2405-2435

23. Peets AD, Cooke L, Wright $B$, Coderre $S$, McLaughlin $\mathrm{K}$ (2010) A prospective randomized trial of content expertise versus process expertise in small group teaching. BMC Med Educ 10:70
24. Cook DA (2010) Twelve tips for evaluating educational programs. Med Teach 32:296-301

25. Bowen JL (2006) Educational strategies to promote clinical diagnostic reasoning. New Engl J Med 355:2217-2225

26. Graber ML, Franklin N, Gordon R (2005) Diagnostic error in internal medicine. Arch Internal Med 165:1493-1499

27. Langewitz W (2011) Patientenzentrierte Kommunikation. In: Adler RH, Herzog W, Joraschky P et al (Hrsg) Uexküll. Psychosomatische Medizin. Theoretische Modelle und klinische Praxis. Elsevier, Urban \& Fischer, München, S 338-347

28. Langewitz WA, Loeb Y, Nubling $M$, Hunziker $S$ (2009) From patient talk to physician notes - comparing the content of medical interviews with medical records in a sample of outpatients in Interna Medicine. Patient Education Couns 76:336-340

29. Back AL, Arnold RM, Baile WF et al (2007) Efficacy of communication skills training for giving bad news and discussing transitions to palliative care. Arch Internal Med 167:453-460

30. Bachmann C, Hölzer H, Dieterich A et al (2009) Longitudinales, bologna-kompatibles Modell-Curriculum „Kommunikative und Soziale Kompetenzen": Ergebnisse eines interdisziplinären Workshops deutschsprachiger medizinischer Fakultäten. GMS Z Med Ausbild 26:Doc38

31. Cegala DJ, Lenzmeier Broz S (2002) Physician communication skills training: a review of theoretical backgrounds, objectives and skills. Med Education 36:1004-1016

32. Duffy FD, Gordon GH, Whelan G et al (2004) Assessing competence in communication and interpersonal skills: the Kalamazoo II report. Academic Medicine: J Assoc Am Medical Colleges 79:495-507

33. Rider EA, Hinrichs MM, Lown BA (2006) A model for communication skills assessment across the undergraduate curriculum. Med Teach 28:e127134

34. Solomon DJ, Szauter K, Rosebraugh CJ, Callaway MR (2000) Global ratings of student performance in a standardized patient examination: Is the whole more than the sum of the parts? Advances Health Sci Education: Theory Practice 5:131-140

35. Kurtz S, Silverman J, Draper J (1998) Teaching and learning communication skills in medicine. Radcliffe Medical Press Ltd., Abingdon Oxon, UK

36. Humphris GM, Kaney S (2001) The Liverpool brief assessment system for communication skills in the making of doctors. Advances Health Sci Education: Theory Practice 6:69-80

37. Hodges B, Hanson M, McNaughton N, Regehr G (2002) Creating, monitoring, and improving a psychiatry OSCE: a guide for faculty. Acad Psychiatry 26:134-161

38. Hodges B, Mcllroy JH (2003) Analytic global OSCE ratings are sensitive to level of training. Medical Education 37:1012-1016

39. Scheffer S, Muehlinghaus I, Froehmel A, Ortwein $H$ (2008) Assessing students' communication skills: validation of a global rating. Advances Health Sci Education: Theory Practice 13:583-592

40. Newble D (2004) Techniques for measuring clinical competence: objective structured clinical examinations. Medical Education 38:199-203

41. Steele DJ, Hulsman RL (2008) Empathy, authenticity, assessment and simulation: a conundrum in search of a solution. Patient Education Couns 71:143-144

42. Wear D, Varley JD (2008) Rituals of verification: the role of simulation in developing and evaluating empathic communication. Patient Education Couns 71:153-156
43. Cherry MG, Fletcher I, O'Sullivan H, Shaw N (2012) What impact do structured educational sessions to increase emotional intelligence have on medical students? BEME Guide No. 17. Med Teach 34:1119

44. Berkhof $M$, van Rijssen $H J$, Schellart AJ, Anema JR, van der Beek AJ (2011) Effective training strategies for teaching communication skills to physicians: an overview of systematic reviews. Patient Education Couns 84:152-162

45. Schildmann J, Kupfer S, Burchardi N, Vollmann $J$ (2012) Teaching and evaluating breaking bad news: a pre-post evaluation study of a teaching intervention for medical students and a comparative analysis of different measurement instruments and raters. Patient Education Couns 86:210-219

46. Dauphinee WD, Wood-Dauphinee S (2004) The need for evidence in medical education: the development of best evidence medical education as an opportunity to inform, guide, and sustain medical education research. Academic Medicine: J Association Am Medical Colleges 79:925-930

47. Cook DA, Beckman TJ, Bordage G (2007) Quality of reporting of experimental studies in medical education: a systematic review. Medical Education 41:737-745

48. Thistlethwaite J, Hammick M (2010) The Best Evidence Medical Education (BEME) Collaboration: into the next decade. Med Teacher 32:880-882

49. Hammick M, Haig A (2007) The Best Evidence Medical Education Collaboration: processes, products and principles. Clin Teach 4:42-45

50. Tamblyn R, Abrahamowicz M, Dauphinee D et al (2007) Physician scores on a national clinical skills examination as predictors of complaints to medical regulatory authorities. JAMA 298:993-1001

51. O'Neill PA, Jones A, Willis SC, McArdle PJ (2003) Does a new undergraduate curriculum based on Tomorrow's Doctors prepare house officers better for their first post? A qualitative study of the views of pre-registration house officers using critical incidents. Medical Education 37:1100-1108

52. Zick A, Granieri M, Makoul G (2007) First-year medical students' assessment of their own communication skills: a video-based, open-ended approach. Patient Education Couns 68:161-166

53. Hulsman RL, Harmsen AB, Fabriek M (2009) Reflective teaching of medical communication skills with DiViDU: assessing the level of student reflection on recorded consultations with simulated patients. Patient Education Couns 74:142-149

54. Lee CA, Chang A, Chou CL, Boscardin C, Hauer KE (2011) Standardized patient-narrated web-based learning modules improve students' communication skills on a high-stakes clinical skills examination. J Gen Internal Med 26:1374-1377

55. Norcini J, Burch V (2007) Workplace-based assessment as an educational tool: AMEE Guide No. 31 . Med Teach 29:855-871

56. Pelgrim EA, Kramer AW, Mokkink HG, van den Elsen L, Grol RP, van der Vleuten CP (2011) In-training assessment using direct observation of single-patient encounters: a literature review. Advances Health Sci Education: Theory Practice 16:131-142 